\title{
Influence de la quantité de matière sèche ingérée et du temps de séjour des aliments sur la digestibilité de la ration par la chèvre laitière
}

\author{
T Najar ${ }^{1}$, S Giger-Reverdin ${ }^{1}$, D Sauvant ${ }^{1}$, C Poncet ${ }^{2}$ \\ 1 Station de Nutrition et Alimentation (INRA) de I'INA-PG, 16, rue C-Bernard, 75231 Paris \\ Cedex 05; 2 Station de Nutrition des Herbivores, INRA, Theix, 63122 Ceyrat, France
}

\begin{abstract}
Summary - Simultaneous measurements of organic matter digestibility (OMD) and particulate mean retention time (MRT) of diets containing various proportions of hay and concentrate (bran or beet pulp) were performed in lactating or dry goats. Beyond the hay and concentrate influences, the $\mathrm{OMD}$ decreased by 0.5 and 1.3 points as the dry matter intake level increased by $10 \mathrm{~g} / \mathrm{kg} \mathrm{LW} 0.75$ and MRT decreased by $5 h$, respectively.
\end{abstract}

Introduction - Najar et al (1988) ont observé que, chez des chèvres présentant des niveaux d'ingestion de matière sèche (MSI) et de production laitière différents, le temps de séjour moyen (TSM) des particules était négativement influencé par la MSI. Dans ce travail, des mesures de digestibilité de la matière organique des régimes $\left(d M O_{r}\right)$ ont été réalisées, afin de savoir si cette caractéristique était spécifiquement influencée par MSI ou TSM.

Matériel et Méthodes - Douze chèvres dont 3 taries et les 9 autres produisant entre 0,5 et $2,8 \mathrm{~kg}$ de lait, sont placées en cages de digestibilité. Elles reçoivent au prorata de leur appétit (niveau de refus $15 \%$ ) une ration constituée de foin de luzerne + dactyle, seul ou associé à du son de blé ou à de la pulpe de betteraves. Les rations mixtes sont offertes en mélange de manière à ce que les proportions fourrages/concentrés soient indépendantes des niveaux de production. Le protocole de mesure de transit, suivant la méthode préconisée par Poncet et al (1987), a été décrit par Najar et al (1988). Des mesures individuelles de $d M O_{r}$ ont été pratiquées, en même temps que celles des transits, pendant 2 périodes d'une semaine, sé- parées de $7 \mathbf{j}$. Les résultats de $d M O_{\text {r }}$ ont été ajustés selon le modèle de régression : $d M O r=$ $d M O_{f} \cdot F+d M O_{p} \cdot P+d M O_{s} \cdot S+a \cdot T S M_{r}$ (ou $M S I$ ), avec $F+P+S=1$, dans lequel $d M O_{f}$ $d M O_{p}$ et $d M O_{s}$ sont respectivement les estimations de la dMO du foin, de la pulpe et du son, sous l'hypothèse que leurs proportions mesurées $F, P$ et $S$ au sein de la MO du régime sont indépendantes. Le TSM moyen de la ration $\left(T S M_{r}\right)$ est calculé en pondérant les TSM mesurés simultanément du fourrage et du concentré par leurs proportions respectives $(F, P$ ou $S$ ). MSl est exprimé en $\mathrm{g} / \mathrm{kg} P V 0,75$. Les variables $T S M_{r}$ et $M S /$ sont centrées pour éviter la présence d'une constante dans l'équation.

Résultats et Discussion - Sur les 24 mesures effectuées, n'ont été prises en compte que les 17 pour lesquelles l'apport des aliments marqués n'avait pas influencé l'ingestion. Le tableau I rapporte les principales caractéristiques des mesures effectuées. Conformément au protocole, il n'y a pas de relation entre la $M S I$ et les proportions $F, P$ et $S$, alors que la $M S /$ est très liée $(R=0,80)$ au niveau de production de lait. La corrélation globale entre $d M O_{\text {r }}$ et $M S I$ est nulle (tableau I), par 
Tableau I. Caractéristiques des variables étudiées.

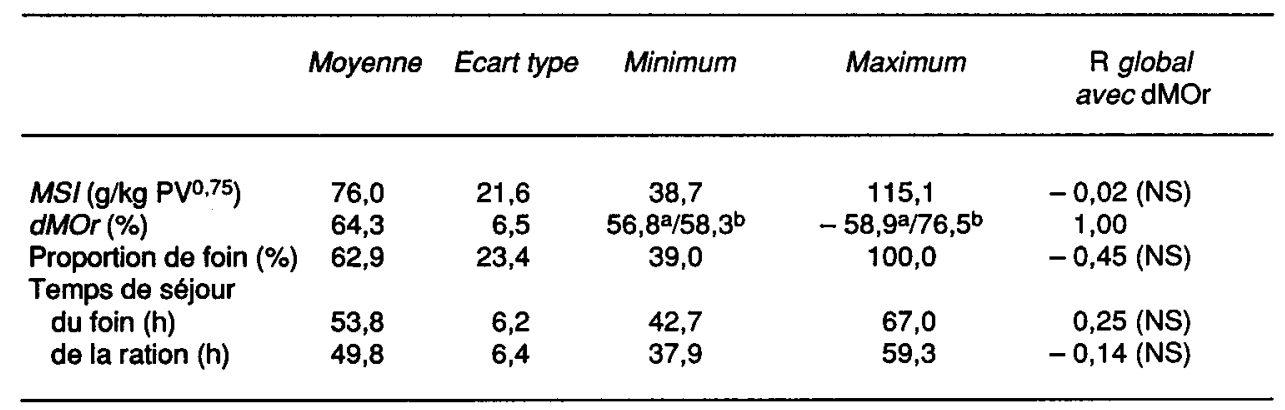

a Rations foin seul. 'b Rations foin + concentré.

contre, compte tenu de l'influence des proportions $F, P$ et $S$ prises en covariables, il apparait que la $d M O_{r}$ baisse significativement de 0,5 points en moyenne, lorsque le niveau de $M S I$ croît de $10 \mathrm{~g} / \mathrm{kg} P V 0,75$ : $d M O r=58,0(0,8) \cdot F+65,3(1,7) \cdot S+85,4$ $(1.6) \cdot P-0,051(0.021) \cdot M S I(E T R=1,83)$.

La prise en compte du $T S M_{r}$ qui ne présente pas de relation globale avec $d M O_{r}$ (tableau 1), aboutit à un ajustement statistiquement meilleur: $d M O_{r}=56,9$ $(0,7) \cdot F+67,1(1.3) \cdot S+87,2(1,3) \cdot P+0,26$ $(0,06) \cdot T S M_{r}(E T R=1,38)$. Les coefficients de cette équation demeurent stables lorsque $T S M_{r}$ est remplacé par celui du foin. La $d M O_{r}$ diminue donc significativement de 1,3 point lorsque le $T S M_{r}$ décroît de $5 \mathrm{~h}$.

L'interprétation des résultats expérimentaux par la méthode des différences aboutit à des valeurs $d M O_{f}, d M O_{p}$ et $d M O_{s}$ de $57,8,66,4$ et $85,0 \%$ respectivement. Ces valeurs ne sont pas différentes de celles fournies par les coefficients des deux équations. Les résidus de ces deux régressions sont étroitement corrélés ( $R=$ $0,80)$ ce qui traduit la relation entre $T S M_{r}$ et $M S I\left(T S M_{r}=65,8-0,211 \cdot M S\right), \quad R=$ $0,71)$ et l'incapacité commune des caractéristiques prises en compte à expliquer cer- taines valeurs de $d M O_{r}$ Une analyse de ces résidus, en fonction des combinaisons de $F, P$ et $S$, ne permet pas de révéler de phénomène d'interaction digestive, par contre, la répétition des mesures permet de mettre en évidence des variations individuelles significatives. Pour la première équation, l'écart type inter-individu est de 1,9 point et sa prise en compte dans la régression aboutit à un ETR de 1,1 point. Pour la seconde, les valeurs correspondantes sont respectivement de 1,4 et 0,8 point. Le $T S M_{r}$ tend donc à mieux intégrer les variations individuelles de $d M O_{r}$ que $M S I$. Les résidus des deux régressions sont moins liés $(P<0,10)$ lorsque ces variations individuelles sont prises en compte.

Une influence spécifique du niveau de $M S I$ et du TSMr sur la digestibilité d'un régime mixte peut donc être dégagée en s'aftranchissant de l'influence des proportions de fourrages et d'aliments concentrés.

Najar T, Giger S, Poncet C, Sauvant D (1988) Reprod Nutr Dev 28, 113-114

Poncet C, Gomez L, Michalet-Doreau B, Geay Y (1987) Reprod Nutr Dev 27, 219-220 